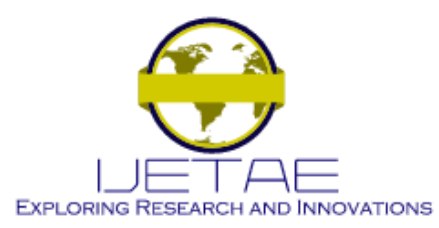

International Journal of Emerging Technology and Advanced Engineering

Website: www.ijetae.com (E-ISSN 2250-2459, SCOPUS Indexed Journal, Volume 10, Issue 12, December 2020)

\title{
A Novel Compact Multi-Axis Force Sensor
}

\author{
Yash Gujarati ${ }^{1}$, Ravindra Thamma ${ }^{2}$ \\ ${ }^{1,2}$ Robotics and Mechatronics Engineering Technology, Central Connecticut State University, USA
}

\begin{abstract}
This paper presents the development of a sixaxis force/torque (FTS) sensor using crossbeams for a robotic arm. The sensor produced in this paper is a new unique design that was developed under rigorous trial and testing using finite element analysis (FEA) at every stage of development. Additionally, the FTS presented uses strain gauge technology and data-acquisition (DAQ) to measure and record forces in $\mathrm{Fx}, \mathrm{Fy}$, and $\mathrm{Fz}$ direction along with torque in Mx, My, and Mz direction. FTS was tested, calibrated, and fitted on a robotic arm to test its accuracy and repeatability.
\end{abstract}

Keywords - Six-axis force/torque sensor, crossbeams, FEA

\section{INTRODUCTION}

Robotic arms play an important role in the current automation industries. They have been used for more than five decades with demand always increasing at a rapid rate. Currently, robotic arms are used in automobile, electronics, packaging, food industries, and many more similar industries that require either repeatability, constant rate of manufacturing, or accuracy. This has also given rise to collaborative robotic arms that can work side by side with humans. With such human-robot interaction, the robotic arms need to be equipped with sensors that can sense the presence of humans and objects around themselves. This is to ensure that there are no damaging accidents between humans and machines. To achieve this force-sensing ability, the robotic arms need to be equipped with what is called a six-axis force/torque sensor (FTS). An FTS is a device that is capable of sensing, recording, regulating force, and torque exerted on the machine [1]. Robotic arm equipped with such FTS allows it to be accepted widely by industries. As such sensors improve human-robot safety, as well as increase production rate at much higher accuracy.

Before moving forward, it should be made clear that to measure force and torque, using a six-axis FTS mounted on the end of a robotic arm is not the only way of measuring forces and torque. Another popular way of measuring forces and torque is to have a joint based sensor. A novel design was proposed by Min, J. K., et al [2], where a reactive-type joint torque sensor was developed. A joint based sensor closely resembles a conventional sensor. But a joint-based sensor is limited to measure torques and poorly performs measuring the forces.
Other available options are link strain gauges, motor current sensors, and flexibility modeling [3]. But all of them lack accuracy, and repeatability when compared to the six-axis force/torque sensor. Thus, conventional FTS is a better choice.

Above said, a robotic arm equipped with an FTS also comes with many benefits such as load feedback, greater precision and sensitivity, collision detection, and highresolution measurements. All these advantages are from the result of FTS capability measure all three components of force, Fx, Fy, and Fz, as well as all three components of torque, Mx, My, and Mz [1]. With the FTS, specific tasks such as deburring, grinding, polishing, mechanical assembly and inspection become much easier to perform at a continuous rate. When performing these tasks, the computer or operator is continuously receiving feedback from the sensor, the robotic arm can be instructed to maintain a constant pressure between the part and the robotic arms end effect of using feedback data [4]. With help of constant pressure, the outcome has a much better finish and a much faster rate of production. This also gives the final output a craftsman-like result where every part is receiving meticulous attention to detail.

Currently, available ready-to-use FTS in the market are very expensive which can cost anywhere starting from $\$ 4,200$ [5] and up to $\$ 10,000$ [6] and only capable of measuring a set range of force and torque. While these readily available sensors in the market do offer all the good attributes of an FTS, most consumers are unfortunately not able to afford them. For those reasons, this paper will focus on designing and producing a much affordable FTS capable of measuring a wider range of force and torque. The FTS produced in this paper uses strain gauge technology to measure the force and torque component. The reason a strain gauge was opted rather than any other available force sensor was its capability of measuring at higher stability for long term measurements, higher precision, and ease of error adjustment [7] compared to other force sensors such as piezoelectric force sensors or force-sensitive resistors (FSRs). But it must be noted strain gauges are much more brittle and susceptible to temperature change. Strain gauges require great attention while being used and handled. 


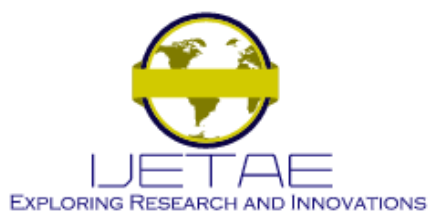

International Journal of Emerging Technology and Advanced Engineering

Website: www.ijetae.com (E-ISSN 2250-2459, SCOPUS Indexed Journal, Volume 10, Issue 12, December 2020)

Before the work of designing the sensor, a ground plan was laid out showing using a flow chart in figure 1. A sixaxis force/torque consists of three major design requirements which are the metal enclosure, the mounted strain gauges, and the circuit inside the enclosure. The resistance change from the strain gauge passes through a series of circuits and instruments before the change in force/torque is visually represented. The process is shown in the flow chart below.

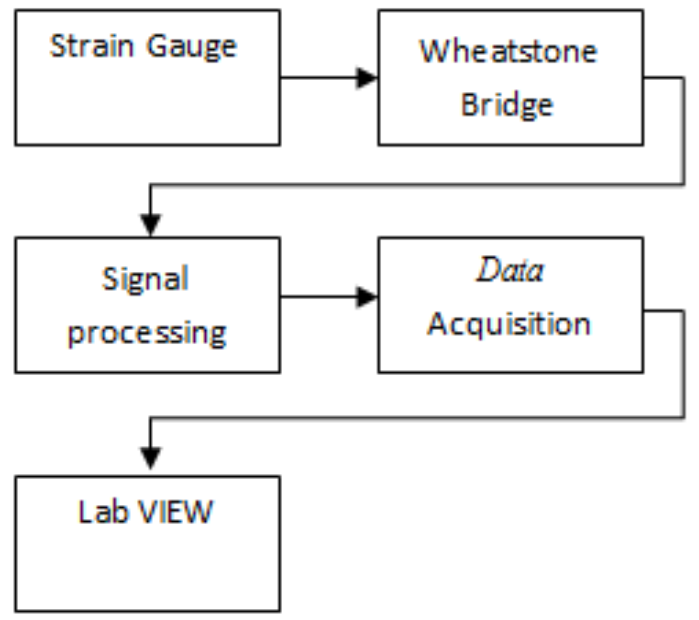

Figure 1. Ground plan for designing six-axis force/torque sensor

\section{LITERATURE REVIEW}

The design of the metal enclosure is the most important part of an FTS. Depending on the requirement, great attention is needed for designing this aspect of the sensor. There are various kinds of six-axis force/torque developed with a different structural design using strain gauges. FTS designed by Ubeda et al [8] made use of hub-sprocket design to reduce production cost and to produce a lightweight sensor using a strain gauge. Chen et al [9] designed a six-axis force/torque sensor with thin flexible cross beams with a hollow center which is quite different from existing sensors. The hole in the center allows cables to pass through as well as to provide a thin cross beam wall for maximum strain measurements. The thin rigid body was manufactured separately and then fitted inside a round enclosure. A unique design proposed by Liang [10] used an upper casing for insulation purposes. With help of the casing, there was very little temperature interference on the strain gauges. The inside of the sensor had an E-type membrane with a small hole in the center to carry wires. Different types of designs presented and discussed by Khan et al [11] show how quickly the design of the enclosure can get complicated.
Example designs are shown in figure 2 to figure 7. Figure 2 represents are simple widely used hollow design. This type of structure is sensitive and is much easier to design, fabricate, and implement. Even though the structure is so simple, the amount of force and the number of axes in which forces and torque can be measured is limited.

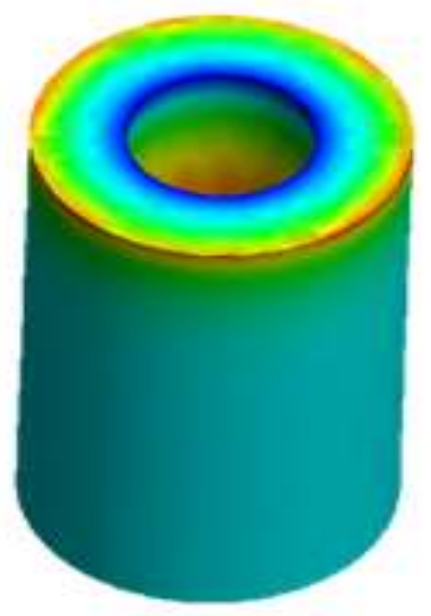

Figure 2. Hollow cylinder design

Figure 3 shows a design that uses the concept of strain gauges, though, as mention in the paper [11], this design has its flaws. Mounting a strain gauge on such a structure is quite difficult and has higher stiffness. The design can be further optimized to increase its sensitivity.

Figure 4 shows a complex hub-sprocket design with a 4 bar linkage. At first glance, this design looks unusual and complex, but it works on the 4-bar linkage principle to measure strain.

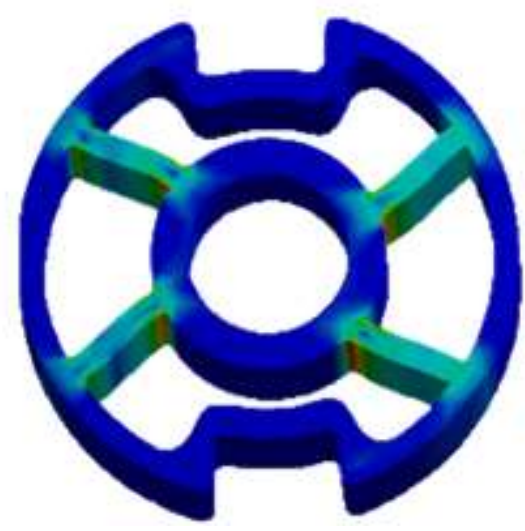

Figure 3. Hub-sprocket with four spokes 


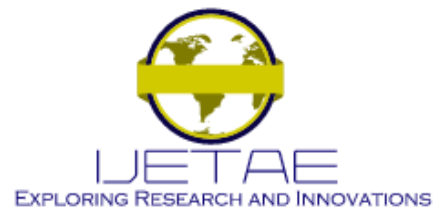

International Journal of Emerging Technology and Advanced Engineering

Website: www.ijetae.com (E-ISSN 2250-2459, SCOPUS Indexed Journal, Volume 10, Issue 12, December 2020)

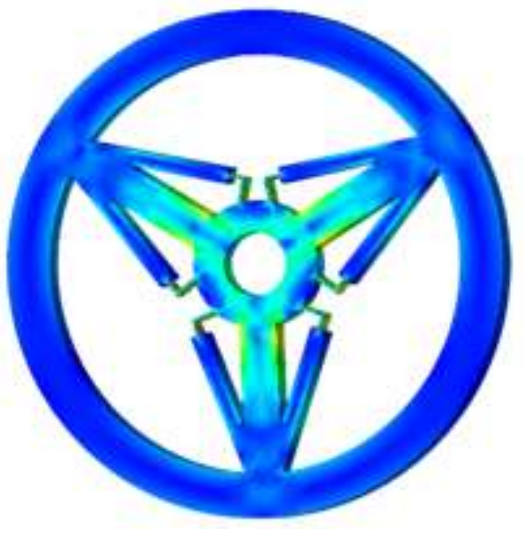

Figure 4. Hub-sprocket with 4-linkage bar

This design is highly sensitive because of the thin walls used here. Unfortunately, this design is susceptible to breakage because of the thin walls and required a high cost of manufacturing.

The hollow hex form design is proposed by Aghili, Farhad, et al [12] in figure 5, is a compact design where the design shape, material properties, and overloading were considered during its designing and fabrication stage. The hollow hex form design also had high stiffness thanks to a high number of wing pairs. On the other hand, such a design structure will surely increase manufacturing cost, and mounting the strain gauges on the wings will also be difficult to perform. The mounting location areas are harder to access.

Figure 6 is the design proposed by Khan et al [11], this design is a solution, which keeps the ease of strain gauge mounting, and high sensitivity in mind.

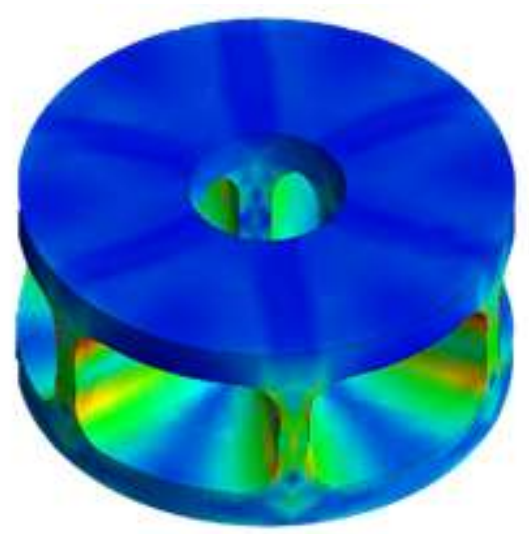

Figure 5. Hollow Hex forms

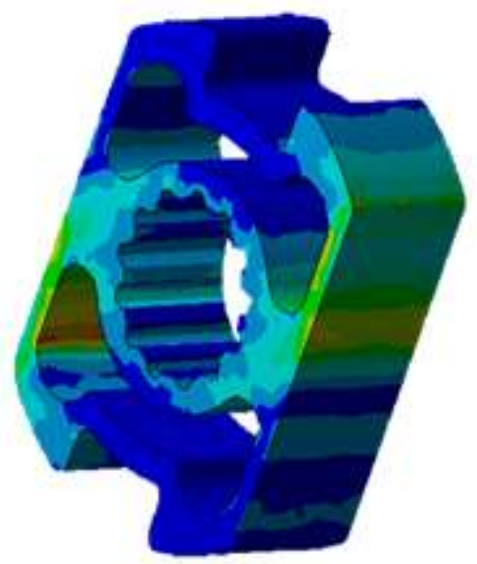

Figure 6. Square-cut torque sensors [9]

Unfortunately, this design is only limited to joint based torque measuring and requires multiple parts which will increase manufacturing cost. From these examples, it is clear how difficult this task was going to be when it comes to enclosure design.

A unique approach by Yao, Jiantao, et al [13], was to use $3 \mathrm{D}$ printed parts to create a parallel force sensor. The material used for 3D printing was titanium alloy. The sensor was designed with a fixed circular platform with eight spoke on the inside. Strain gauges were mounted on these thin spokes all connected to a central platform. Using $3 \mathrm{D}$ printed parts for making a six-axis force/torque sensor has not been done before. Moreover, their paper concludes with promising results with less than $0.71 \%$ error in force measurement and less than $2.37 \%$ in torque measurements in the z-axis. Such unique approaches will surely promote research in a novel design using $3 \mathrm{D}$ printing.

Another non-conventional design approach was presented by S. Shams, J. Y. Lee, and C. Han [14], where a compact and lightweight sensor was designed using a photo-interrupter sensor rather than a strain gauge. Their design made use of five spokes connected to a central base. During the design stage, they also performed multiple FEA tests on the strain measuring part to test the design. Kim [15][16][17], developed multiple six-axis force/torque sensors for an intelligent robot, robotics arm gripper, and robotic intelligent foot. All those designs used what is called parallel plates. Kim's design made use of parallel plates, beams, and strain gauges to measure forces and torques. 


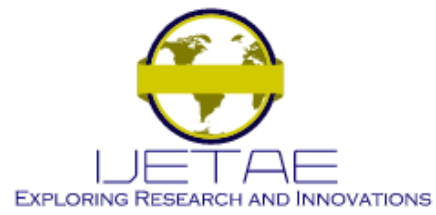

International Journal of Emerging Technology and Advanced Engineering

Website: www.ijetae.com (E-ISSN 2250-2459, SCOPUS Indexed Journal, Volume 10, Issue 12, December 2020)

For strain gauge technologies, data acquisitions, and data processing, the papers by Gujarati, Thamma, and Laber [1] [18], presents currently available technologies available for FTS and their demands. The paper talks about the increasing demand for six-axis force/torque sensor and how most of the available FTS are very expensive. The two papers also present and compare technologies available for strain measuring, signal processing, and data acquisitions. From the presented technologies, the most reliable choices for strain measuring presented were strain gauges. For signal amplification, Wheatstone bridges were said to be the consistent approach. The paper talks in-depth about the types of Wheatstone bridge, and how to use them. Also compares strain gauges, piezoelectric force sensors, and force-sensitive resistors.

Above said, the overall structure of the FTS proposed in this paper had few requirements: The sensor enclosure was required to be as sleek as possible with very few moving parts. The structure was needed to be capable of measuring a wide range of strain changes. The enclosure is also required to be lightweight; this issue was addressed by building the data processing unit on the outside of the sensor. A unique design is also proposed in the paper to make the sensor body sleek and compact by using no moving parts and making use of beam bending. From the above literature review, some of the design concepts were sort of combined to come up with a design structure that would overcome the issue of mounting strain gauge and to make the sensor highly sensitive. The idea of four beam hub-sprockets from figure 3 and a stiff design structure of hollow hex form from figure 5 was adapted. From two of these designs, an antibody stiff structure was developed.

\section{DESIGN APPROACH}

The sensor being an elastic part, the enclosure required trial and error of the designs in finite element analysis (FEA). For those reasons, the metal enclosure was designed in Solid Works computer-aided design (CAD) software and countless stress and strain analyses at various loads were performed. With help of the CAD software, the strain measuring capability can be tested of a design without having to manufacture it. This saves a lot of time and resources. From the literature review, it was clear that the enclosure design needed to be circular to fit well on a robotic arm. On the inside, most of the designs from the literature review used beams of different shapes and sizes. A beam design is quite simple and works on the beam bending principle. When force is exerted on a beam, the atoms in the material will try to oppose the force and try not to break under the load.
The bending of the material is the behavior response from the beam. Overloading will cause the material to break, but when used below its calculated limit, the beam will bend and will also return to its original shape. This property of the beam structure makes the shape an ideal solution for measuring strain.

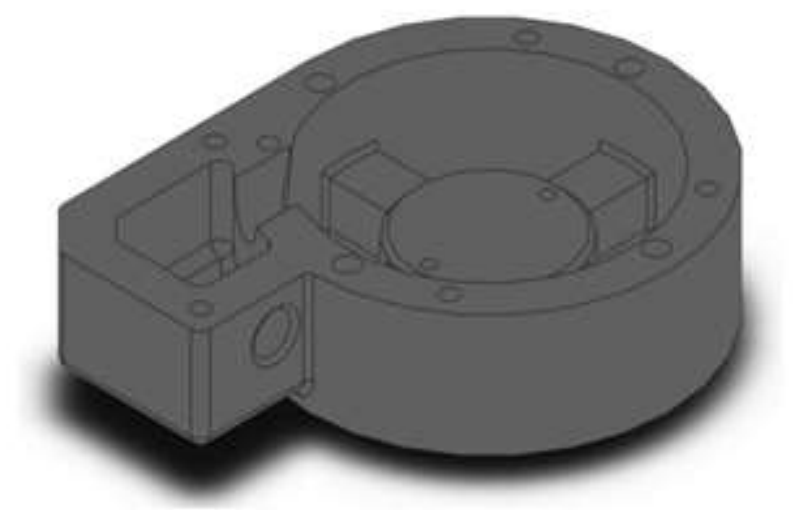

Figure 7. Initial design for FTS with side extrusion for cable/circuit management

Initially, the design was made of a round enclosure with a small box extrusion on the side to make room for the electronics circuits and cables. This is shown in figure 7. This design did show promising results, however produced uneven deformation on the beam used in the design. This was an issue and would produce incorrect results. The side with the extrusion had a more rigid structure and resulted in reduced deformation when compared to sides without an extrusion. Though this design would have produced an FTS with lower height and a slimmer body. Nevertheless, the design with side extrusion was entirely removed for a perfect round design.

\section{A. Design}

After examining multiple designs by various researchers, it was decided to have an antibody circular enclosure fabricated out of a single solid piece of metal shown in figure 8 . With this approach, the cost of manufacturing is lowered by a huge margin. Using a single piece of metal to form the structure meant lowering the cost of production. Only the use of a lathe machine and milling machine was required to fabricate the bottom structure shown in figure 8 . As can be seen in the figure, the strain sensing beams are milled out of the base of the sensor. With such a design, all the force/torque experienced by the body was directly transferred to the beams. This type of beam design is called a crossbeam elastic body. 


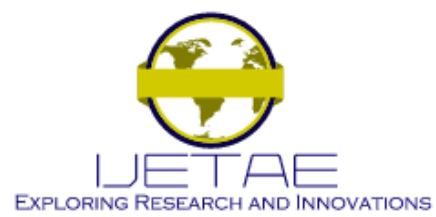

International Journal of Emerging Technology and Advanced Engineering

Website: www.ijetae.com (E-ISSN 2250-2459, SCOPUS Indexed Journal, Volume 10, Issue 12, December 2020)

Most of the designs examined from other papers made use of a hollow circular cylindrical design consisting of thin beams attached to the inside of the cylinder.

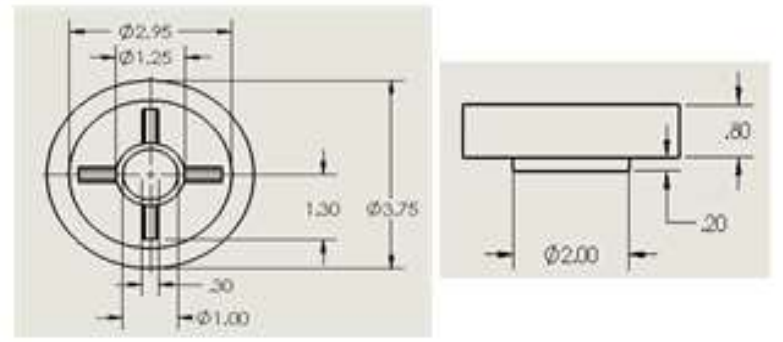

Figure 8. Dimensions for final six-axis force/torque sensor enclosure using cross-beams

Many designs also used sprockets on the inside, or cruciform presented in the paper by Khan et al. unfortunately, these designs are fragile and susceptible to breaking when the sensor is accidentally overloaded.

Moreover, these designs used multiple layers of parts. This can lead to a higher manufacturing cost. For those reasons, a combination of these designs was opted to achieve the advantages of multiple designs. The metal enclosure has a thin base which allows the deformation to transfer from the enclosure base to the beams on which the strain gauges are mounted. The thickness of the base is roughly $1 / 16$ of an inch thick. With such thin walls, the deformation is guaranteed transferred to the beams.

When forces in Fx, Fy, or Fz directions are applied, the beams deform in those respective directions, the strain gauges mounted of the beams thus experience tension or compression. With multiple strain gauges in various locations on the beams, the sensitivity increase. The locations were determined to be on the top and sidewalls of the beams. For the exact locations, FEA analysis was conducted which showed the best location for the strain gauges to be mounted.

\section{B. Metal Selection}

Selecting a metal type plays an important role in the sensor. Different materials have different metal properties such as different density, tensile strength, modulus of elasticity, and Poisson's ratio. Each of these properties of different metals must be examined and compared [18]. When selecting metals, there are two obvious choices, stainless steel, and aluminum, the reason being both the metals have a higher modulus of elasticity, and tensile strength. Moreover, aluminum is a very lightweight material, and weight does play an important role when designing a six-axis FTS.
When selecting the right kind of stainless steel or aluminum alloy, few factors must consider. The metal enclosure must have a high yield limit, the material should be widely available, excellent balance of strength and weight, corrosion resistance, and easy to machine. Table 1.0 provided below compares widely used metal for sensor enclosure.

TABLE I

PARAMETERS OF METALS FOR COMPARISON

\begin{tabular}{|l|l|l|l|}
\hline \multicolumn{1}{|c|}{ Metal } & $\begin{array}{c}\text { Density } \\
\text { (g/cc) }\end{array}$ & $\begin{array}{c}\text { Tensile } \\
\text { strength } \\
\text { (MPa) }\end{array}$ & $\begin{array}{c}\text { Modulus of } \\
\text { Elasticity } \\
\text { (Gpa) }\end{array}$ \\
\hline Aluminum 2024 & 2.78 & 469 & 73.1 \\
\hline Aluminum 6061 & 2.7 & 310 & 68.9 \\
\hline $\begin{array}{l}\text { Aluminum 7075 - } \\
\text { T6 }\end{array}$ & 2.81 & 572 & 71.7 \\
\hline 347H Stainless steel & 7.96 & 515 & 193 \\
\hline $\begin{array}{l}\text { 17-4PH Stainless } \\
\text { steel }\end{array}$ & 7.75 & 1170 & 196 \\
\hline
\end{tabular}

Ubeda, et al [8], used aluminum 7075 - T6 for their design due to the metal's ease of machinability, high tensile strength, modulus of elasticity, and low density. The aluminum alloy does cost slightly higher than other alloys, however, its advantages offset the price. Paper by Sun, Yongjun, et al [29], used alloy 17-4PH stainless steel for its high modulus of elasticity, high yield strength, and high tensile strength. 17-4PH has a much higher yield strength and tensile strength than needed for the design proposed in this paper. At such high tensile strength, the amount of deformation is lower and harder to detect. Thus, a material with lower tensile strength was required. Aluminum alloy 2024 and alloy 6061 are also two other metals that have been used by manufacturers and researchers for a low-cost, lightweight sensor. Unfortunately, they lack the high tensile strength needed for measuring high tension and deformation. Then there is alloy steel $347 \mathrm{H}$ [19] which was specifically developed for enhanced creep resistance, meaning the metal will not deform easily under continuous loading, unloading, or when force is applied for a long period. Alloy $347 \mathrm{H}$ has adequate Tensile strength, modulus of elasticity, and density. A benefit of using steel over aluminum is its capability of supporting high tension forces and good safety factor against overloading. 


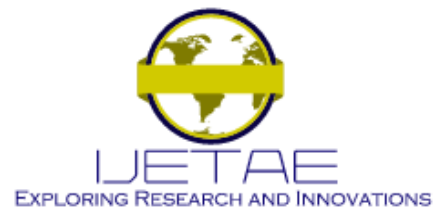

International Journal of Emerging Technology and Advanced Engineering

Website: www.ijetae.com (E-ISSN 2250-2459, SCOPUS Indexed Journal, Volume 10, Issue 12, December 2020)

Finally, the material is well suited for high temperature works and is excellent corrosion resistant.

Alloy $347 \mathrm{H}$ is less machinable compared to other material presented in the table except for $17-4 \mathrm{PH}$, which is also harder to cut or mill. $347 \mathrm{H}$ is also denser material, which is compensated by a compact design. The benefits certainly outweigh the less machinability and high density. All these properties were fulfilling the requirements.

\section{FEA Analysis}

The CAD software used to design the enclosure can perform FEA analysis at various user-specified loads. Once the metal for the enclosure is selected, an FEA analysis on the design can be performed. For the analysis, the simulation tool provided in Solid Works was used. First, a virtual fixture is decided, followed by a load of userspecified weight, then the direction of the force is chosen or the torque, and finally the simulation is performed by the computer. From the FEA analysis, the maximum deformation on the beam is right at the center. The von Misses at the center of the beam under load in the $\mathrm{x}$ and $\mathrm{y}$ direction was found to be $116.089 \mathrm{MPa}$ and deformation of $319.7 \mu \varepsilon$. An even force of $10 \mathrm{~N}$ was applied to each FEA analysis.

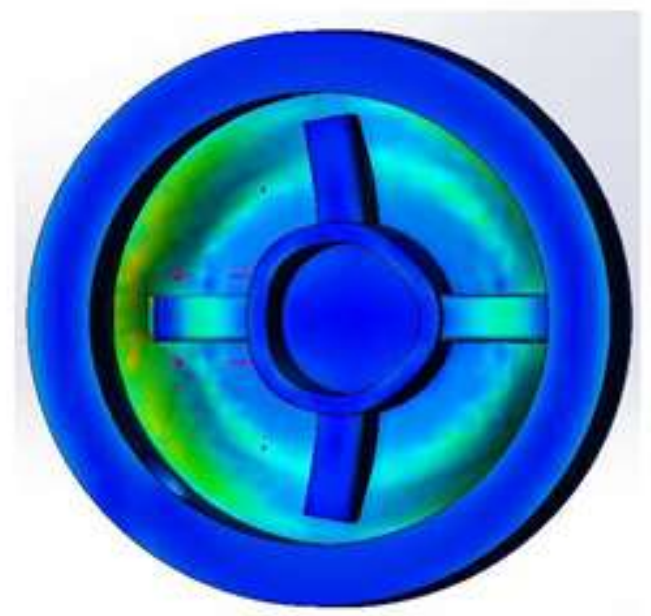

Figure 9. FEA analysis, force applied in $x$-axis from left to right of the page

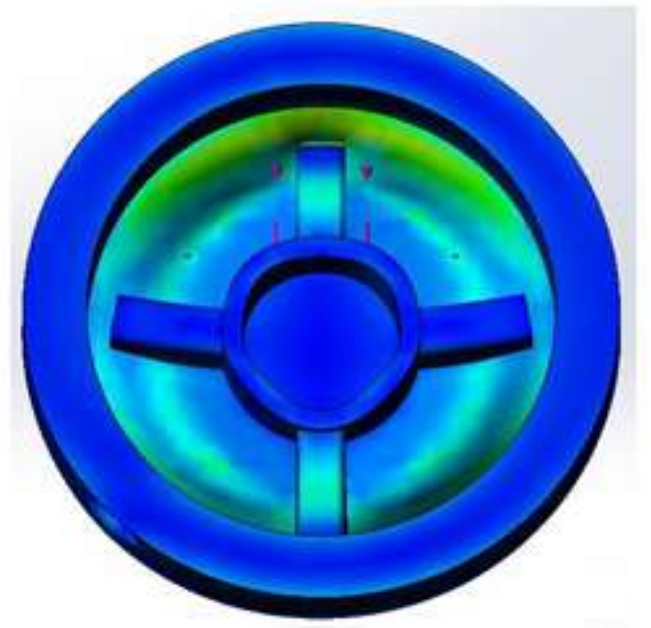

Figure 10. FEA analyses, force applied in y-axis from top to bottom of the page

In figure 9 is can be seen how the top and bottom beams are in compression whereas the left and right beams are in tension, hinting a force being applied in the x-direction, specifically from left to right. The same can be said for figure 10 where the force is applied in the y-direction, from top to bottom. Though when looking at figure 11, the analysis shows all beams are in tension at the center suggesting the applied is in the $\mathrm{z}$-direction.

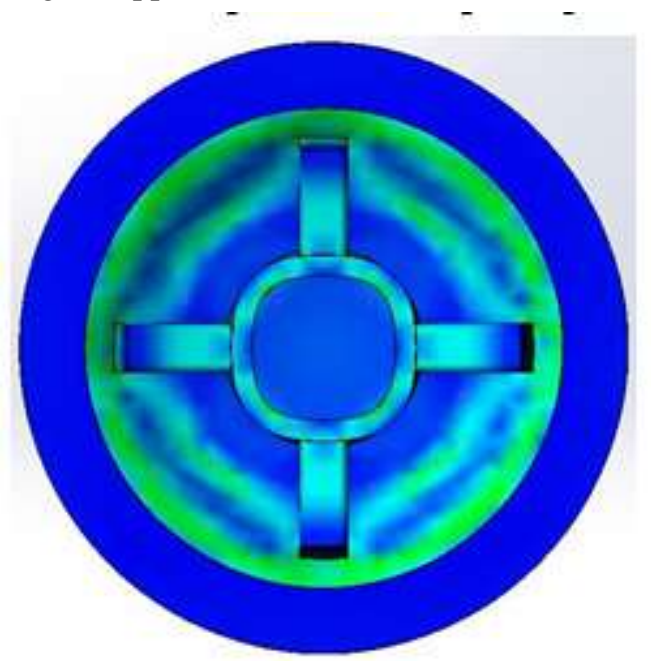

Figure 11. FEA analyses, force applied in z-axis, into to the paper 


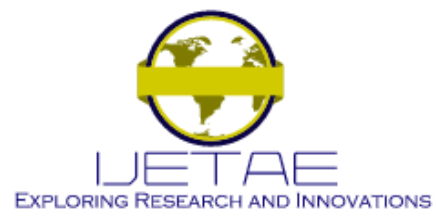

International Journal of Emerging Technology and Advanced Engineering

Website: www.ijetae.com (E-ISSN 2250-2459, SCOPUS Indexed Journal, Volume 10, Issue 12, December 2020)

Finally, when the sensor was experiencing torque in counterclockwise in figure 12, the sidewalls of the beams experienced maximum tension on the wall facing away from the direction of the torque.

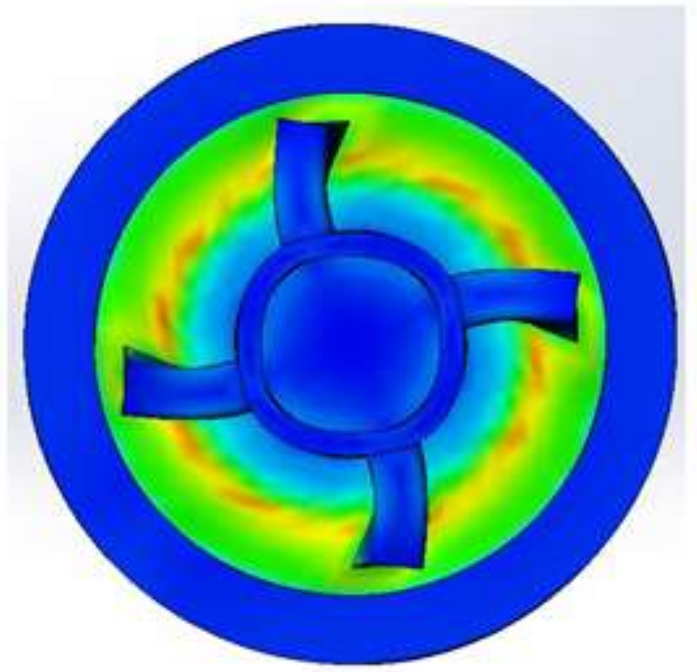

Figure 12. FEA analyses, torque applied in counterclockwise

The beam wall facing towards the direction of the torque experience compression. With help of tension and compression data, the direction of the torque can be found. For maximum deformation, the design went through multiple changes, and at each stage, FEA analysis was done to better understand the design. From the analysis, the optimal location for the strain gauge was also determined, which was at the center of the beam where maximum deformation was observed.

\section{Arrangement of Strain Gauges}

Once the maximum deformation location was found using FEA analysis, the strain gauge mounting location was decided. Two strain gauges were mounted perpendicular to each other on the top surface of each beam and two strain gauges on each side of the beam placed parallel, totaling 6 strain gauges on each beam. The 4 strain gauges on the side made a full bridge, with this configuration maximum bending strain can be measure. All the strain gauge locations are shown in figure 13; strain gauges are colored in yellow. The two gauges placed perpendicular on the top side of the beam created a half-bridge, using this configuration, the axial strain can be measured. Strain gauges used are SGD-1.5/120-LY11 from Omega [20]. These strain gauges are linear $120 \mathrm{ohms}$ strain gauges with a tolerance of $0.5 \%$ and a gauge factor of 2 . The strain gauge also relatively small, sizing $4.70 \mathrm{~mm} \times 3.4 \mathrm{~mm}$.
The strain gauges are arranged in a full-bridge configuration as shown in figure 14 along with its equation; this type of circuit layout is called rhombus style. Some of the reasons test engineers prefer Wheatstone bridges over any other methods are more accurate measurements compared to laboratory millimeters, capable of measuring a very small change in resistance, temperature compensation is possible, measurement sensitivity increases greatly when using multiple beams are used at the same time. All the reasons were the requirements, such as the smallest change in force was needed from the FTS, temperature compensation, and the design was already using multiple beams. Thus, a Wheatstone bridge was used over any other available methods. Moreover, using a Wheatstone bridge is economical as no other components were required.

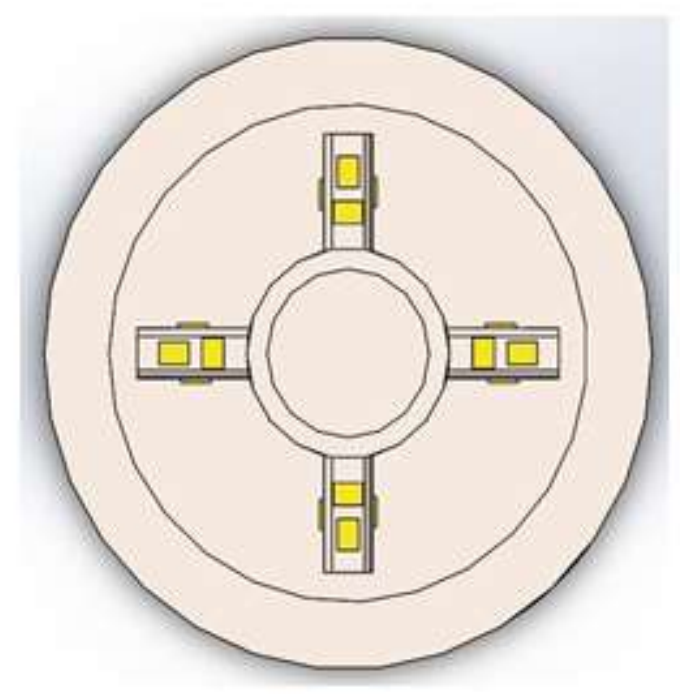

Figure 13. Strain gauge mounting locations of the beams.

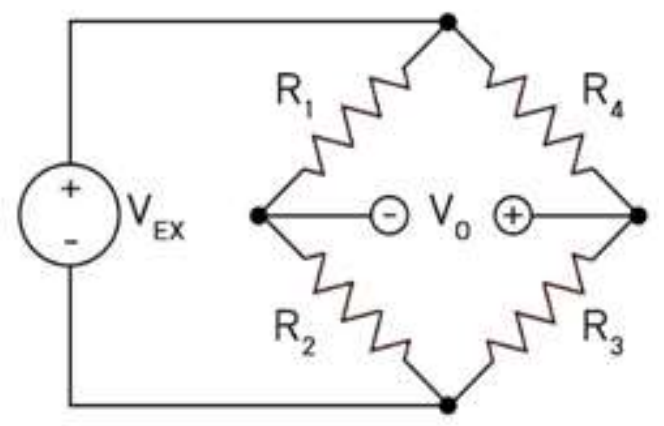

Figure 14. Wheatstone bridge circuit configurations in rhombus style [21] 


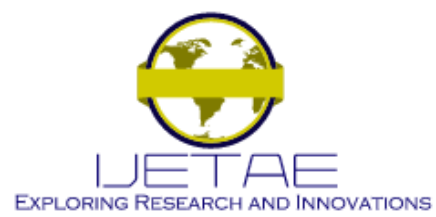

International Journal of Emerging Technology and Advanced Engineering

Website: www.ijetae.com (E-ISSN 2250-2459, SCOPUS Indexed Journal, Volume 10, Issue 12, December 2020)

$$
\begin{gathered}
V_{O}=\left[\frac{R_{3}}{R_{3}+R_{4}}-\frac{R_{2}}{R_{2+} R_{1}}\right] * V_{E X} \\
V_{O}=\left[\frac{R_{3}}{R_{3}+R_{4}}-\frac{R_{2}}{R_{2+} R_{1}}\right] * V_{E X}
\end{gathered}
$$

Eq.(1)

The gauges on the top of the beams form a half-bridge if used by it. This means two more resistors of $120 \mathrm{ohms}$ will be required to build a full bridge, which can produce errors in results. So, four gauges from two beams are combined to form a second full bridge. In total, there are six full bridges. The circuit diagram for six Wheatstone bridges is shown in figure 15. The circuit diagram was designed in National Instruments Multiuser software. Alphabets marked from A$F$ represent the junctions where the voltage was measured. In figure 15, A-D Wheatstone bridges measure the strain change on the walls of the cross beams, whereas the separate Wheatstone bridges $\mathrm{E}$ and $\mathrm{F}$ measure the strain from the strain gauges mounted on top of the cross beams.
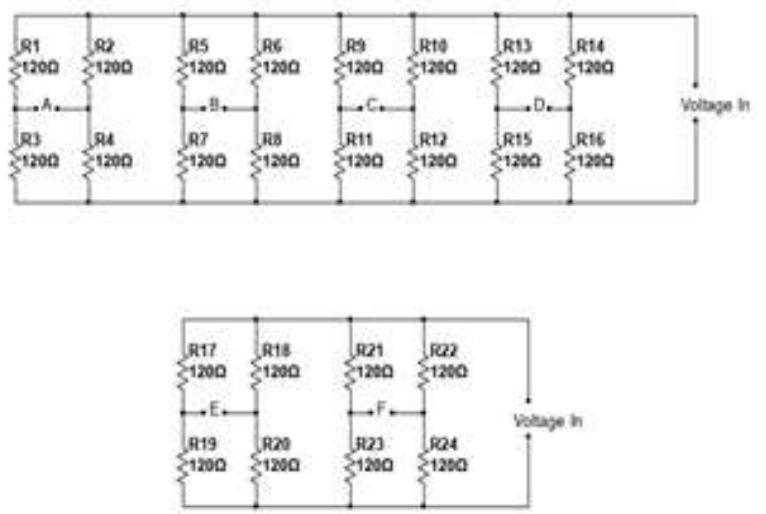

Figure 15. Final strain gauge circuit configurations

\section{E. Design Equations and Calculation}

Calculating the strain from the beam is important as the calculated strain value must be compared to the measured value during calibration to confirm that the sensor is functioning as predicted. The calculations are derived from beam bending and deformation, specifically, Timoshenko beam theory [22][23] [24] is used. The cross beams are deflecting $(\omega) \omega)$ and have a rotational angle $(\varphi(\varphi)$. The Timoshenko beam theory equations are the following:

$$
M(x)=-E I \frac{d \varphi(x)}{d x} M(x)=-E I \frac{d \varphi(x)}{d x}
$$

$$
\begin{gathered}
F_{Q}(x)=k G A\left(\frac{d \omega(x)}{d x}-\varphi(x)\right) \\
F_{Q}(x)=k G A\left(\frac{d \omega(x)}{d x}-\varphi(x)\right)
\end{gathered}
$$

From the equation, the deflection and rotation angle equations at the center of the beams are obtained [25]:

$$
\begin{gathered}
\omega(x)=-\frac{F l^{2} x}{16 E I}+\frac{F x^{3}}{12 E I}-\frac{F x}{2 k G A} \\
\omega(x)=-\frac{F l^{2} x}{16 E I}+\frac{F x^{3}}{12 E I}-\frac{F x}{2 k G A} \\
\varphi(x)=-\frac{F l^{2}}{16 E I}+\frac{F x^{2}}{4 E I}-\frac{F x}{2 k G A} \\
\varphi(x)=-\frac{F l^{2}}{16 E I}+\frac{F x^{2}}{4 E I}-\frac{F x}{2 k G A}
\end{gathered}
$$

From the above two equations, the equation for the direct strain can be obtained and its further derivative.

$$
\begin{aligned}
& \varepsilon(x, z)=-z \frac{d \varphi(x)}{d x} \varepsilon(x, z)=-z \frac{d \varphi(x)}{d x} \\
& \varepsilon(x, z)=\frac{F x z}{2 E I} \varepsilon(x, z)=\frac{F x z}{2 E I}
\end{aligned}
$$

Derivation of the above equation and substituting $\mathrm{I}=$ $\mathrm{b}^{3} \mathrm{~h} / 12$, we get

$$
\varepsilon(x, z)=\frac{3 F l}{2 E b^{3}} \varepsilon(x, z)=\frac{3 F l}{2 E b^{3}}
$$

It is important to know that these equations work for force and moment on the beam at the center of the beam. The final equation 8 is

\section{F. 3-D Printing}

Before making a part of solid steel $347 \mathrm{H}$, the design was $3 \mathrm{~d}$ printed for visual inspection and to examine the final design. The reason $3 \mathrm{~d}$ printing was opted at this stage was to increase speed, lower cost, higher flexibility, risk reduction, and sustainability. With help of $3 \mathrm{~d}$ printing, the final product can be observed and examined. 


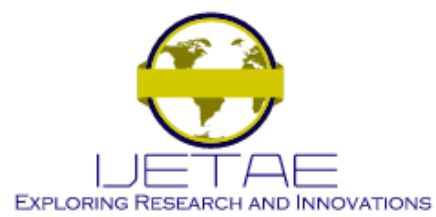

International Journal of Emerging Technology and Advanced Engineering

Website: www.ijetae.com (E-ISSN 2250-2459, SCOPUS Indexed Journal, Volume 10, Issue 12, December 2020)

Compared to making the part out of steel, the $3 \mathrm{~d}$ printing only took $1 / 10$ the time. $3 \mathrm{~d}$ printing lowers production costs and reduces the risk of fabricating an incorrect part.

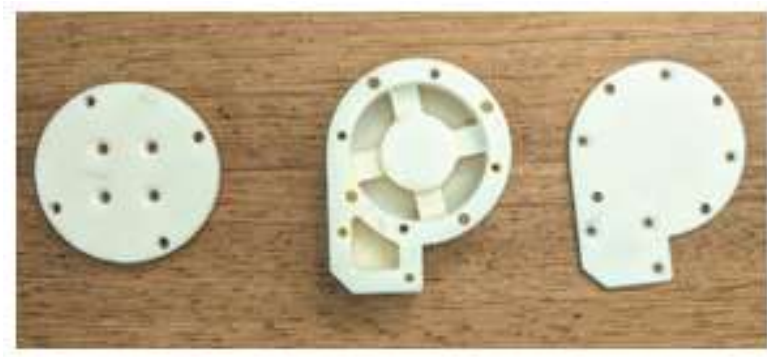

Figure 16. 3D printed parts produced before final fabrication

For instance, early-stage $3 \mathrm{~d}$ printing revealed the initial mounting whole placements were placed at the wrong location. If the incorrect mounting holes were made in the final part, the material cost and time it took to fabricate the part will go to waste. Figure 16 shows the 3d-printed parts. The below figures are of the initial enclosure design which was later discarded for a better design.

\section{G. Manufacturing Process}

The manufacturing of the part was performed in-house using a few easily available machines. First, a stock of alloy steel $347 \mathrm{H}$ measuring 4 " in diameter was cut to a size of 1.25 " height using an electric saw. The part was then loaded onto a lathe machine and was the necessary turning cuts were made from the top. $347 \mathrm{H}$ being a hardened metal requires slow speed cutting using a high-speed steel cutter. A constant stream of cutting oil was flown over the part during the entire process of turning. A slow feed of $0.5 \mathrm{~mm} / \mathrm{t}$ was maintained for cutting with pauses in between to cool down the part and cutting tool. Once the initial turning was done in the lathe, the part was then loaded onto a milling machine for final cutting. In the mill, the conditions were kept similar to the lather cutting process and the final cutting was performed. Mounting holes on the part were done using drill machines.

Given the $347 \mathrm{H}$ metal is slightly difficult to cut compared to other steel alloys and aluminum, the result is well worth the effort. For large-scale production, the process can be done using a $\mathrm{CNC}$ machine and the manufacturing time can be reduced drastically. Though for this paper, only a few test pieces were required, and thus the process was done manually in-house.

\section{StRain CALCULATION}

The output from the strain gauge is a very small amount of resistance change.
This change in resistance is in micro-ohms which are quite difficult to measure and record, even some of the most expensive lab instruments are not capable of measuring the small change in resistance with ease. For those reasons, an amplifying circuit capable of amplifying and converting this small change to something measurable is needed. Thus, a Wheatstone bridge is used here. A Wheatstone bridge converts the small change in resistance from the strain gauges to a measurable amount of voltage change in mill volts. It is equivalent to two parallel voltage dividers using four resistors. A strain gauge is essentially a resistor; thus, a Wheatstone bridge is well suited for the task. Using equation 1 for a Wheatstone bridge, the voltage output can be measured. However, equation 1 needs to be modified when using 4 similar strain gauges in parallel. Since the 4 strain gauges are mounted on the sidewalls of the beams, they act as a cantilever beam, where two gauges are in compression and the other two in tension. This induces both a negative and positive change in resistance. Thus, the following equation is used for calculating strain with a known load, voltage, and resistance.

$$
\begin{gathered}
V_{O}=\frac{V_{E X}}{4} *\left[\frac{\Delta R_{1}}{R_{1}}-\frac{\Delta R_{2}}{R_{2}}+\frac{\Delta R_{3}}{R_{3}}-\frac{\Delta R_{4}}{R_{4}}\right] \\
V_{O}=\frac{V_{E X}}{4} *\left[\frac{\Delta R_{1}}{R_{1}}-\frac{\Delta R_{2}}{R_{2}}+\frac{\Delta R_{3}}{R_{3}}-\frac{\Delta R_{4}}{R_{4}}\right] \\
V_{O}=\frac{V_{E X}}{4} * \frac{k}{4}[\varepsilon 1-\varepsilon 2+\varepsilon 3-\varepsilon 4] \\
V_{O}=\frac{V_{E X}}{4} * \frac{k}{4}[\varepsilon 1-\varepsilon 2+\varepsilon 3-\varepsilon 4]
\end{gathered}
$$

In the above equation, the R1, and R4 are under tension, while R2 and R3 are in compression. The same equation is used for all four beams. For the gauges mounted on top of the beam, a different equation is needed as their orientation is different.

$$
\begin{aligned}
& \varepsilon=\frac{1}{2(1+v)} * \frac{4}{k} * \frac{V_{O}}{V_{E X}} \\
& \varepsilon=\frac{1}{2(1+v)} * \frac{4}{k} * \frac{V_{O}}{V_{E X}}
\end{aligned}
$$

In all of the above equation, the $V_{O} V_{O}$ is voltage input, $V_{E X} V_{E X}$ is voltage being measured, $v v$ is Poisson's ratio, $\boldsymbol{k} \boldsymbol{k}$ is gage factor which is 2 for all the strain gauges used. The equation for top strain gauges is repeated for each gauge. 


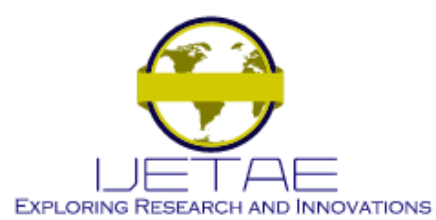

\section{International Journal of Emerging Technology and Advanced Engineering Website: www.ijetae.com (E-ISSN 2250-2459, SCOPUS Indexed Journal, Volume 10, Issue 12, December 2020)}

\section{DAta ACQuisition And Signal Processing}

The data output from Wheatstone bridge is in mill volts between the ranges of $10 \mathrm{mV}$ to $100 \mathrm{mV}$, this is a measurable amount using a lab multimeter, though amplified voltage would yield better results. To amplify the voltage, there are a few available options. Such as building an operational amplifier to amplify the voltage. Though there are ICs available which are dedicated to amplifying voltage from a Wheatstone bridge? HX711 [26] is a 24-bit ADC IC available at a fraction of cost compared to building an Op-Amp. The IC is capable of 128 gains. To put this into perspective, a 10-bit microcontroller such as at mega 328P can amplify and measure the voltage at a rate of 1024 steps. At such step count, voltage changes of $4.9 \mathrm{mV}$ can be detected. Unfortunately, that is a large step, at such resolution; the weight being measured has very low resolution. On the other hand, HX711 has a 16,777,216step count. This means a voltage change of 0.298 microvolts can be measure. At such a small step, even a penny coin can be weighed. Though it must be taken into consideration, with such small steps, there is more noise being measure alongside weight change. Fortunately, the noise can be eliminated during the signal processing stage.

A single HX711 IC can only facilitate one Wheatstone bridge, usually, a combinatory board is used for combining up to four Wheatstone bridge, but that will lower the resolution of measured weight. Thus, six HX711 ICs were used in total for measurements.

Once the voltage from the Wheatstone bridge is amplified the voltage change needs to be processed where unwanted noise is removed, calibration is performed, and the data is collected for visual representation. For the signal processing, Compact DAQ by National Instruments (NI) [27] was used. Before using the DAQ, the application development environment and NI-DAQ mix drive software were installed provided by NI.

When using NI-DAQ mix comes preloaded with taskspecific architecture, such as frequency, temperature, voltage, strain measurements, etc. Strain measurement architecture is loaded, followed by physical channel selections to which the input signals are connected. Once the hardware part of the signal processing is done, the remaining steps are done in the software. In the software, the expected maximum and minimum strain values are entered, the gage factor, strain gauge resistance, initial voltage, source voltage, lead wire resistance, and finally strain configuration are also entered.
The DAQ software by NI will provide a circuit representation of how all the connections must be done before calibration is performed. Even shunt resistors of offset resistor values can be entered, though this was not done as no extra resistors were used.

Once all the data is entered, the software will present all the values which are measured value, error, gain adjustment, simulated strain, and measured strain. These values are presented to compare with calculated data before finally being visually represented in graphical form.

\section{CALIBRATION PROCESS}

A calibration process is very important to produce an FTS that works and performs similarly to the calculated amount. At this stage, all the calculated and measured data from the sensor were entered into a table for comparison. There are various methods to perform the task of calibration, such as setting up a calibration bench as done by Ubeda et al [8]. Kim et al [28] performed a similar experimental calibration where weight on the sensor was hung from a jig to exert known weights on a mini force/torque sensor. For this paper, the physical sensor was subjected to a weight of $10 \mathrm{~N}$ in the direction of Fx, Fy, and Fz. The forces are then measured using the sensor's strain gauges, circuit, and DAQ. The output from the Lab View is then observed and verified if the force being measured is $10 \mathrm{~N}$. The steps are then followed again at increments of 10 $\mathrm{N}$ for up to $100 \mathrm{~N}$. All the data calculated and measured were well within $3.4 \%$ error.

Once the forces in Fx, Fy, and Fz are measured and compared, the sensor was then subjected to varying torque. Both in the clockwise direction and anti-clockwise direction. Again, calculated and measured values are compared and a similar error to $3.4 \%$ was found. Once all the data was calculated, measured, and compared, changes were made to the program for minor adjustments. These adjustments allow us to calibrate the sensor through software change rather than physical change. If the sensor is not performing as expected, the second option would be to rearrange the strain gauges, add dummy resistors, or make major changes to the circuit. That would certainly be time-consuming. And given the strain gauges are permanently mounted on the beams, removing, and trying to mount them back would certainly make the gauges unusable. Thus, software-based calibration is preferred here. 


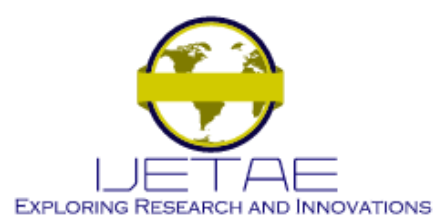

\section{International Journal of Emerging Technology and Advanced Engineering Website: www.ijetae.com (E-ISSN 2250-2459, SCOPUS Indexed Journal, Volume 10, Issue 12, December 2020)}

Finally, the sensor was subjected to absurdly large forces to see its performance under accidental overloading. The sensor reached its limit at over $600 \mathrm{~N}$ where the error was well over $11 \%$. At this point, even software calibration would not work all the time as intended and cause measuring wrong forces and torques.

\section{EXPERIMENTAL IMPLEMENTATION}

Once all the calibrations and tests were performed, the sensor was installed on a robotic arm. This is where final calibration and tests were performed. Given the FTS was not a factory-installed part and rather a user added part, the end effector on the arm needed to be offset through the arm controller. Then the arm was tasked to a simple pick and place jobs. Where various weights were programmed to be picked and packed at the constant velocity over a flat surface. Sort of mimicking the palatalization process. All the weights being lifted and being placed down were labeled with their weights in grams and Newton's. When the weights were being lifted, the robotic arm process was paused at its maximum height for two seconds to record the data. The data recorded from the FTS at this point was compared to the actual weight of the pieces being moved around.

A similar calibration test was carried out for measuring torque, where the arm was tasked to insert a screw in a solid piece of metal. In this test, the side forces were not needed to be measured. The main outcome of this calibration test was to only measure torque in clockwise and anti-clockwise. For a final test, all six parameters of a robotic arm were tested and compared to a commercial sixaxis force/torque sensor.

\section{RECOMMENDATION FOR FUTURE STUDY}

The six-axis force/torque sensor proposed in this paper can still undergo many design improvements and changes for a much better result with much less error. Some of the improvements which can be made to the sensor are the enclosure design improvements and signal processing unit improvements.

Starting with the enclosure design, for future improvements, the design proposed by Sun, Yongjun, et al [29] [30] makes use of a strain measuring plate of a sort which is a separate body placed inside as sensor base.

Another similar design for an enclosure where a separate strain measuring body is used for measuring force is proposed by D. Okumura, S. Sakaino, and T. Tsuji [31]. Their design uses a high-rigidity flexure element and a lowrigidity flexure element.
By using two separate measuring elements, forces as small as $0.01 \mathrm{~N}$ and up to $1000 \mathrm{~N}$ of force was measured with almost zero error. The sensor proposed is not made for a robotic arm, but a similar concept can be applied to a sixaxis FTS. With such a design structure, the strain sensing body can be designed and manufactured separately. By manufacturing and installing the parts separately, the sensitivity of the sensor increases greatly. Mounting of strain gauge on the unit also becomes easier. A similar approach can be made for future improvements.

For signal processing, either a dedicated signal processing unit capable of measuring ever so small change in resistance from strain gauge can be purchased or an inhouse build op-amp circuit can be designed for better noise reduction, signal amplification, and real-time selfcalibration. A circuit, wire, or component through which the current is flowing will always be susceptible to noise. With 24 strain gauges and double the number of wires used in the design proposed, there is still a small amount of noise even after passing the voltage through a DAQ system capable of eliminating noise. Thus, for future improvement, a dedicated noise filter circuit can be designed to eliminate the remaining noise.

Another improvement that can be made to the design is the addition of the Ethernet/ethernetIP or PROFINET interface. By connecting the FTS to a network, easy remote operating and monitoring can be done. Data from the sensor can easily be sent to various departments in a manufacturing plant if needed without any hassle [32].

\section{CONCLUSION}

In this paper, a six-axis force/torque sensor was successfully developed using cross-beams with less than $3.4 \%$ error in measurement. The design was optimized using FEA and 3-printing. Compared to traditional design, the cross-beam designed was proven to be sensitive, and much easier to manufacture and implement in a robotic arm. A circuit design for measuring the strain change and an effective signal processing solution was also presented. This was done using an IC chip capable of amplifying the output voltage from the Wheatstone bridge and passing the output from the IC into a DAQ system. Finally, the FTS was calibrated and tested where all six components for force and torque; Fx, Fy, Fz, and Mx, My, Mz respectively, were successfully measured.

Although the paper verified and presented a successful FTS design, in future work, a much more optimized design can be created with less than $1 \%$ error. 


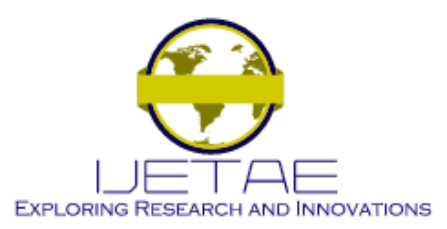

\section{International Journal of Emerging Technology and Advanced Engineering Website: www.ijetae.com (E-ISSN 2250-2459, SCOPUS Indexed Journal, Volume 10, Issue 12, December 2020)}

\section{REFERENCES}

[1] Gujarati, Yash, and Ravindra Thamma. "Survey of Force/Torque Sensor for Industrial Robotic Arm." International Journal of Innovative Science, Engineering \& Technology, vol. 7, no. 11, Nov. 2020, ijiset.com. 2

[2] Min, J. K., Ahn, K. H., Park, H. C., \& Song, J-B. (2019). A novel reactive-type joint torque sensor with high tensional stiffness for robot applications. Mechatronics, 63, [102265]. https://doi.org/10.1016/j.mechatronics.2019.102265

[3] Helmick, D., et al. "A Comparison of Force Sensing Techniques for Planetary Manipulation.” 2006 IEEE Aerospace Conference, 27 Oct. 2005, doi:10.1109/aero.2006.1655724

[4] Tian, Fengjie, et al. "Polishing Pressure Investigations of Robot Automatic Polishing on Curved Surfaces." The International Journal of Advanced Manufacturing Technology, vol. 87, no. 1-4, 2016, pp. 639-646., doi:10.1007/s00170-016-8527-2.

[5] “On Robot HEX-E - High Precision Force/Torque Sensor." Thinkbot Solutions LLC, thinkbotsolutions.com/products/onrobot-hexe?variant $=31674599145552$. [Last accessed: $12 / 05 / 2020$ ]

[6] "On Robot RG2-FT - Smart Gripper with Force/Torque Sensor." Thinkbot Solutions LLC, thinkbotsolutions.com/products/onrobotrg2-ft?variant=31678532747344. [Last accessed: 12/05/2020]

[7] T. Kleckers "Force sensors for strain gauge and piezoelectric crystalbased mechatronic systems- a comparison," 2012 IEEE International Instrumentation and Measurement Technology Conference Proceedings, Graz, 2012, pp. 2306-2308, DOI: 10.1109/I2MTC.2012.6229515.

[8] Ubeda, Rodrigo Perez, et al. "Design and Manufacturing of an UltraLow-Cost Custom Torque Sensor for Robotics.” Sensors, vol. 18, no. 6, 2018, p. 1786. Doi: 10.3390/s18061786.

[9] Chen, Danfeng, et al. "Design and Calibration of a Six-Axis Force/Torque Sensor with Large Measurement Range Used for the Space Manipulator.” Procedia Engineering, vol. 99, 2015, pp. 1164 1170. doi:10.1016/j.proeng.2014.12.699.

[10] Liang, Qiaokang, et al. "Design and Fabrication of a SixDimensional Wrist Force/Torque Sensor Based on E-Type Membranes Compared to Cross Beams." Measurement, vol. 43, no. 10, 2010, pp. 1702-1719., doi:10.1016/j.measurement.2010.09.010.

[11] Khan, Hamza, et al. "Towards Scalable Strain Gauge-Based Joint Torque Sensors." Sensors, vol. 17, no. 8, 2017, p. 1905. Doi: 10.3390/s17081905.

[12] Aghili, Farhad, et al. "Design of a Hollow Hex form Torque Sensor for Robot Joints." The International Journal of Robotics Research, vol. 20, no. 12, 2001, pp. 967-976., doi:10.1177/02783640122068227.

[13] Yao, Jiantao, et al. "A 3-D Printed Redundant Six-Component Force Sensor with Eight Parallel Limbs." Sensors and Actuators A: Physical, vol. 247, 2016, pp. 90-97., doi:10.1016/j.sna.2016.05.041.

[14] S. Shams, J. Y. Lee, and C. Han, "Compact and lightweight optical torque sensor for robots with increased range," Sensors and Actuators A: Physical, vol. 173, no. 1, pp. 81-89, 2012.

[15] G. Kim, H. Shin and J. Yoon, "Development of 6-axis Force/Moment Sensor for Humanoid Robot's Foot," SENSORS, 2007 IEEE, Atlanta, GA, 2007, pp. 217-220, DOI: 10.1109/ICSENS.2007.4388375.

[16] Kim, Gab-Soon. (2007). Design of a six-axis wrist force/moment sensor using FEM and its fabrication for an intelligent robot. Sensors and Actuators A: Physical. 133. 27-34. 10.1016/j.sna.2006.03.038.
[17] G. Kim, H. Shin and J. Yoon, "Development of 6-axis Force/Moment Sensor for Humanoid Robot's Foot," SENSORS, 2007 IEEE, Atlanta, GA, 2007, pp. 217-220, DOI: 10.1109/ICSENS.2007.4388375.

[18] Gujarati, Yash, et al. "Technologies for Force/Torque Sensor." International Journal of Innovative Science, Engineering \&amp; Technology, vol. 7, no. 12, Dec. 2020.

[19] Sandmeyer, Steel Company. "Alloy 347/347H Heat Resistant Stainless-Steel Plate." Sandmeyer Steel, www.sandmeyersteel.com/347-347H.html.

[20] Omega. Omega Engineering, 28 Oct. 2016, www.omega.com/enus/force-strain-measurement/strain-gauges/linear-strain-gauges/sgdlinear1-axis/p/SGD-1-5-120-LY11. [Last accessed: 12/05/2020]

[21] All about Circuits. "Strain Gauges: Electrical Instrumentation Signals: Electronics Textbook." All About Circuits, www.allaboutcircuits.com/textbook/direct-current/chpt-9/straingauges/.

[22] S. P. Timoshenko, "On the transverse vibrations of bars of uniform cross-section", London Edinburgh Dublin Philos. Mag. J. Sci., vol. 43, pp. 125-131, Jan. 1922.

[23] Ma, J.; Song, A. Fast Estimation of Strains for Cross-Beams SixAxis Force/Torque Sensors by Mechanical Modeling. Sensors 2013, 13, 6669-6686.

[24] L. Fu, A. Song and D. Chen, "A Polyetheretherketone Six-Axis Force/Torque Sensor," in IEEE Access, vol. 7, pp. 105391-105401, 2019, doi: 10.1109/ACCESS.2019.2932387.

[25] W. Ke F. Du and X. Zhang "Algorithm and experiments of sixdimensional force/torque dynamic measurements based on a Stewart platform" Chin. J. Aeronaut. vol. 29 no. 6 pp. 1840-1851 Dec. 2016

[26] "HX711 Converter. Datasheet Pdf. Equivalent." HX711 Converter Datasheet Pdf - Analog-to-Digital Converter. Equivalent, Catalog, datasheetspdf.com/pdf/842201/A via semi conductor/HX711/1. [Last accessed: $12 / 05 / 2020]$

[27] National Instruments. "Connecting Strain Gages to a DAQ Device." Connecting Strain Gages to a DAQ Device - National Instruments, www.ni.com/getting-started/set-up-hardware/data-acquisition/straingages. [Last accessed: 12/05/2020]

[28] Kim, Y.-G., Kwak, J.-H., Hong, D.-H., \& an, J. (2014). Miniaturized Force-Torque Sensor Built in a Robot End-Effecter for Delicate Tool-Tip Gripping Control. Electronics Ir Elektrotechnika, 20(6), 37. https://doi.org/10.5755/j01.eee.20.6.7258

[29] Sun, Yongjun, et al. "Design and Optimization of a Novel Six-Axis Force/Torque Sensor for Space Robot.” Measurement, vol. 65, 2015, pp. 135-148., doi:10.1016/j.measurement.2015.01.005.

[30] Sun, Yongjun, et al. "Design of a Novel Six-Axis Force/Torque Sensor Based on Strain Gauges by Finite Element Method." Proceeding of the 11th World Congress on Intelligent Control and Automation, 2014, doi:10.1109/wcica.2014.7053277.

[31] D. Okumura, S. Sakaino and T. Tsuji, "High Dynamic Range Sensing by a Multistage Six-Axis Force Sensor with Stopper Mechanism," 2018 IEEE International Conference on Robotics and Automation (ICRA), Brisbane, QLD, 2018, pp. 4065-4070, doi: 10.1109/ICRA.2018.8460571.

[32] Automation, ATI Industrial. ATI Industrial Automation: Net F/T, www.ati-ia.com/products/ft/ft_netft.aspx. [Last accessed: $12 / 05 / 2020$ 